\title{
Lidil
}

Revue de linguistique et de didactique des langues

$58 \mid 2018$

L'enseignement et l'apprentissage de l'écrit académique à l'aide de corpus numériques

\section{Nathalie Lacelle, Jean-François Boutin et Monique Lebrun, La littératie médiatique multimodale appliquée en contexte numérique - LMM@. Outils conceptuels et didactiques}

Presses de l'Université du Québec, 2017, 316 p.

Thierry Soubrié

\section{CpenEdition}

Édition électronique

URL : http://journals.openedition.org/lidil/5217

DOI : $10.4000 /$ lidil.5217

ISSN : 1960-6052

Éditeur

UGA Éditions/Université Grenoble Alpes

Édition imprimée

ISBN : 978-2-37747-064-8

ISSN : $1146-6480$

Référence électronique

Thierry Soubrié, « Nathalie Lacelle, Jean-François Boutin et Monique Lebrun, La littératie médiatique multimodale appliquée en contexte numérique - LMM@. Outils conceptuels et didactiques », Lidil [En ligne], 58 | 2018, mis en ligne le 02 novembre 2018, consulté le 25 septembre 2020. URL : http:// journals.openedition.org/lidil/5217 ; DOI : https://doi.org/10.4000/lidil.5217

Ce document a été généré automatiquement le 25 septembre 2020.

(c) Lidil 


\title{
Nathalie Lacelle, Jean-François Boutin et Monique Lebrun, La littératie médiatique multimodale appliquée en contexte numérique - LMM@.Outils conceptuels et didactiques
}

Presses de l'Université du Québec, 2017, 316 p.

\author{
Thierry Soubrié
}

\section{RÉFÉRENCE}

Nathalie Lacelle, Jean-François Boutin et Monique Lebrun, La littératie médiatique multimodale appliquée en contexte numérique - LMM@. Outils conceptuels et didactiques, Presses de l'Université du Québec, 2017, 316 p.

1 Le présent ouvrage est le second publié par Nathalie Lacelle, Monique Lebrun et JeanFrançois Boutin portant sur la littératie médiatique multimodale. Il s'intéresse exclusivement à la littératie en contexte numérique et cette fois-ci, les auteurs ont pris en charge l'écriture de l'ensemble des chapitres. Le projet éditorial est double puisqu'il s'agit à la fois d'inciter les enseignants à accorder une place aux "pratiques de communication contemporaine en classe » (p. 4), mais aussi de justifier et de légitimer un domaine de recherche, en l'occurrence la littératie médiatique multimodale appliquée en contexte numérique - LMM@.

Dans le premier chapitre, les auteurs situent la littératie numérique au sein des humanités numériques qui succèdent selon eux, dans l'ordre des humanités tel qu'il a été défini par Lévi-Strauss (1973), à l'humanisme démocratique du xxe siècle, comme 
pour mieux signifier sa singularité par rapport à la littératie dite "traditionnelle " (p.65). On retiendra entre autres, bien que le propos ne soit pas central dans ce chapitre, la distinction qui est faite entre, d'une part, les «jeunes» qui ont des pratiques numériques informelles et, d'autre part, l'institution éducative qui résiste à la " culture numérique » (p. 24). Le chapitre suivant est consacré aux genres numériques et à ce qui fait leur spécificité par rapport aux genres préexistants. L'entreprise est intéressante, mais si certaines désignations correspondent à ce que l'on entend par genre, à savoir des «formes abstraites, relativement stables, [...] liés aux différents domaines de l'activité humaine qui en déterminent le contenu thématique, le style et la structure " (Détrie et coll., 2001), à l'instar des fanfictions ou de la narration numérique, d'autres en revanche correspondent davantage à des supports de production (blogue, wiki, etc.) qui, s'ils possèdent des caractéristiques techniques et éditoriales spécifiques, peuvent "accueillir " plusieurs genres. C'est le cas du blogue par exemple qui peut donner lieu à des genres aussi différents que le récit de voyage, le journal intime, ou encore le carnet de recherche.

3 Les chapitres 3 et 4 abordent respectivement la lecture et l'écriture numériques, entre autres envisagées sous l'angle de la multimodalité. À nouveau, il s'agit ici pour les auteurs de montrer, à partir de synthèses de recherches, ce qui fait des activités de lecture, d'écriture et de recherche d'information dans un environnement numérique, des activités différentes et surtout, complexes, qui nécessitent un apprentissage spécifique. Les deux chapitres qui suivent abordent successivement les questions liées à la recherche et à la pédagogie, alors que dans les chapitres précédents les deux dimensions étaient étroitement liées. Dans le chapitre consacré à la recherche, il s'agit de faire le point sur les différentes travaux entrepris jusqu'ici sur la littératie numérique, et ce, dans différents types de recherches (recherche action, recherche ethnographique, design based research, recherche théorique, etc.), et de proposer des pistes pour de futures recherches. Dans le chapitre sur les "design didactiques» (séquence didactique), sont présentés, rapidement, des principes méthodologiques de conception ainsi que "huit séquences d'enseignement de compétences en LMM [littératie médiatique multimodale]» (p. 201).

4 Ce qui fait la force de l'ouvrage, c'est la fusion des propos scientifique et pédagogique qui fournit à travers des exemples de pratiques, certes expérimentales et confidentielles, des idées d'activités à mener en classe. Mais c'est aussi peut-être ce qui fait la faiblesse de l'entreprise. Viser simultanément plusieurs objectifs - partager une conviction, convaincre du bienfondé de pratiques didactiques, asseoir la légitimité d'un objet de recherche, proposer des clés, des pistes pour l'action, faire évoluer les pratiques des enseignants - est très ambitieux et ne permet pas toujours d'offrir au lecteur une vision cohésive de l'ensemble. La publication de cet ouvrage, unique dans la littérature de recherche francophone, témoigne de la détermination d'un groupe de recherche rassemblé autour d'une conviction commune, celle de l'émergence d'une nouvelle littératie et de l'urgence qu'il y a pour l'école à s'en emparer. 


\section{AUTEURS}

THIERRY SOUBRIÉ

LIDILEM, Université Grenoble Alpes 